WellBeing International

WBI Studies Repository

$9-2006$

\title{
Report of the Working Group on Animal Distress in the Laboratory
}

\author{
Marilyn Brown \\ Charles River Laboratories Foundation \\ Larry Carbone \\ University of California - San Francisco \\ Kathleen Conlee \\ The Humane Society of the United States \\ Marian Dawkins \\ University of Oxford \\ Ian J. Duncan \\ University of Guelph
}

See next page for additional authors

Follow this and additional works at: https://www.wellbeingintlstudiesrepository.org/acwp_lab

Part of the Animal Experimentation and Research Commons, Animal Studies Commons, and the Bioethics and Medical Ethics Commons

\section{Recommended Citation}

Brown, M., Carbone, L., Conlee, K. M., Dawkins, M. S., Duncan, I. J., Fraser, D., ... \& Wuerbel, H. (2006).

Report of the Working Group on Animal Distress in the Laboratory. Lab animal, 35(8), 26-30.

This material is brought to you for free and open access by WellBeing International. It has been accepted for inclusion by an authorized administrator of the WBI Studies Repository. For more information, please contact wbisr-info@wellbeingintl.org.

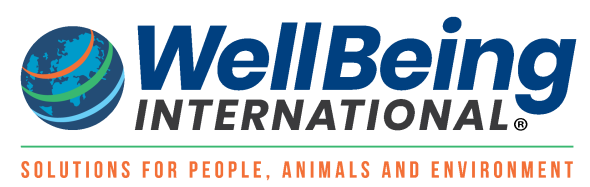




\section{Authors}

Marilyn Brown, Larry Carbone, Kathleen Conlee, Marian Dawkins, Ian J. Duncan, David Fraser, Gilly Griffin, Victoria A. Hampshire, Lesley A. Lambert, Joy A. Mench, David Morton, Jon Richmond, Bernard E. Rollin, Andrew N. Rowan, Martin L. Stephens, and Hanno Würbel 


\section{Report of the Working Group on Animal Distress in the Laboratory}

Marilyn Brown, DVM, MS

Charles River Laboratories Foundation

Larry Carbone, DVM, PhD

University of California - San Francisco

Kathleen M. Conlee, BS

The Humane Society of the United States

Marian S. Dawkins, PhD, MA

University of Oxford

Ian J. Duncan, PhD, BSc

University of Guelph

David Fraser, $\mathrm{PhD}$

University of British Columbia

Gilly Griffin, PhD

Canadian Council on Animal Care

Victoria A. Hampshire, VMD

Food and Drug Administration
Lesley A. Lambert, PhD

Oxford University

Joy A. Mench, D.Phil.

University of California - Davis

David Morton, BVSc, PhD

University of Birmingham

Jon Richmond, MD

Animals in Scientific Procedures

Bernard E. Rollin, PhD

Colorado State University

Andrew N. Rowan, DPhil.

The Humane Society of the United States

Martin L. Stephens, PhD

The Humane Society of the United States

Hanno Würbel, PhD

University of Giessen

\section{CITATION}

Brown, M., Carbone, L., Conlee, K. M., Dawkins, M. S., Duncan, I. J., Fraser, D., ... \& Wuerbel, H. (2006). Report of the Working Group on Animal Distress in the Laboratory. Lab Animal, 35(8), 26-30.

\footnotetext{
ABSTRACT

Finding ways to minimize pain and distress in research animals is a continuing goal in the laboratory animal research field. Pain and distress, however, are not synonymous, and often measures that alleviate one do not affect the other. Here, the authors provide a summary of a meeting held in February 2004 that focused on distress in laboratory animals. They discuss the difficulties associated with defining 'distress,' propose methods to aid in recognizing and alleviating distressful conditions, and provide recommendations for animal research conduct and oversight that would minimize distress experienced by laboratory animals.
} 
This report is the result of a 3-day international expert working group convened in Baltimore, MD on 1113 February 2004. The working group consisted of those listed as authors as well as one participant who chose to remain anonymous and did not sign this report. The working group members, who participated independently, have specialized knowledge and experience regarding animal distress in the laboratory. A number of observers were also present, but did not participate in the formal discussions. The meeting included brief presentations by some of the working group members as well as extensive in-depth discussions.

\section{BACKGROUND}

1. The United States Department of Agriculture (USDA) regulates animal use in research, teaching, and testing (hereafter referred to collectively as 'research') under the Animal Welfare Act, which requires researchers to minimize the 'pain and distress' experienced by research animals. The USDA defines a 'painful procedure' as "any procedure that would reasonably be expected to cause more than slight or momentary pain and/or distress in a human being to which that procedure is applied"1. The USDA has no separate definition for 'distressful procedure'; thus all aspects of distress currently must be encompassed within the regulatory concept of 'pain.' This situation provides impetus for a separate definition that accurately and comprehensively describes distress and/or distressful procedures. The USDA proposed to define distress in research animals in July 2000 (ref. 2), but the proposal proved to be controversial and, as yet, only limited progress on a definition of 'distress' has been made.

2. The ongoing lack of a definition hinders progress toward a comprehensive consideration of laboratory animal distress in the United States, including its recognition, assessment, alleviation, and prevention.

The regulation of animal research focuses on animal pain and distress, acknowledging that these are 'costs' imposed upon the animal which are of public, scientific, and political concern. Opinion polls of the public and other stakeholders, including scientists, indicate that support for animal research declines significantly when the animals are perceived to experience moderate to severe pain and distress (see refs. 3-6). In countries where predicted (prospective) pain, distress, or comparable states are reported, it appears that from one-quarter to one-third of laboratory animals may be at risk for moderate to severe pain or distress ${ }^{7}$.

3. The research community is expected, by both the public and regulatory bodies, to minimize the occurrence and intensity of animal pain and distress. Minimization of pain and distress is important, not only because of the impact on animal welfare, but also because the physiological and psychological effects of pain and distress on animals can compromise research data and conclusions. Consequently, all parties have a stake in identifying and reducing the pain and distress of laboratory animals.

4. The workshop summarized here brought together international scientists and experts in the study of animal welfare and laboratory animal science with the purpose of determining the current state of our understanding of animal distress. The workshop participants identified a number of key issues and future developments, summarized below, that might prove beneficial for scientists, technicians, regulators, and members of animal welfare oversight committees in their continued effort to minimize and prevent laboratory animal distress.

\section{DEFINING DISTRESS}

5. Defining 'distress' is fraught with difficulties. The term has different meanings in different contexts. In an everyday context, 'distress' generally describes a significant unpleasant state experienced by an animal. 
When incorporated into regulatory and legislative language, it is used to establish standards and assess compliance. Finally, in a scientific context, 'distress' should be defined in a way that allows for clear recognition, quantification, and assessment. These three different contexts create a challenge for anybody wishing to produce a definition of distress that is meaningful and practical for operational interpretation by scientists, regulators, and the general public.

6. The difficulty in producing an adequate definition could be addressed by crafting a general description of what might constitute 'animal distress' that is supported by a set of specific examples that provide information about procedures, procedural endpoints, or situations that might be expected to produce different types and levels of distress.

For example, one description, adapted and expanded at the present workshop from wording used in the National Research Council's Guidelines for the Care and Use of Mammals in Neuroscience and Behavioral Research ${ }^{8}$, identifies 'distress' as encompassing:

a range of negative psychological states, such as fear, pain, malaise, anxiety, frustration, depression and boredom that are sometimes associated with exposure to stressors. Distress can be either transient or prolonged and can range from mild to severe, dependent upon the duration and intensity of exposure to the stimulus. Factors such as the species type, cognitive capability, developmental history and individual differences may influence the impact of potentially distressing stimuli.

7. Distress might therefore be considered as one or more negative psychological states indicative of poor well-being and diminishing to an animal's well-being and quality of life. Distress is a concern whenever its extent is more than momentary or slight. Distress is not necessarily related to, nor a consequence of, pain. The nature of an animal's distressful experience can vary widely and may represent states analogous to (but not necessarily exactly the same as) negative human states, such as anxiety, boredom, conflict, depression, fear, frustration, hunger, social isolation and loneliness, malaise, nausea, threat, thirst, and pain. Distress is therefore multi-modal and its extent can vary depending on both the duration and intensity of the stimulus causing distress, as well as the context in which it occurs. It is possible that, due to species differences, there are states of distress in animals that we may not, at this time, be able to identify.

TABLE 1. Categories of possible causes of animal distress in the laboratory

\begin{tabular}{|ll|}
\hline Category of distress source & Example \\
\hline Experimental Treatment & $\begin{array}{l}\text { Infection with pathogen, exposure to fear stimulus (e.g. } \\
\text { predator species), exposure to toxic agent }\end{array}$ \\
\hline Protocol-related procedures & $\begin{array}{l}\text { Restricted feeding, tail biopsy for genotyping, ear } \\
\text { clipping, restraint }\end{array}$ \\
\hline Housing conditions & $\begin{array}{l}\text { Cages with only basic resources, single housing, } \\
\text { lighting type }\end{array}$ \\
\hline Management procedures & Cage cleaning, handling \\
\hline Genetic factors & $\begin{array}{l}\text { Phenotypic expression of pathology in a transgenic or } \\
\text { artificially selected strain }\end{array}$ \\
\hline
\end{tabular}




\section{CAUSATION, RECOGNITION, ASSESSMENT, AND ALLEVIATION OF DISTRESS}

8. Distress can arise from housing animals in physical and social environments that do not support the animals' requirements. One example is the need in mice to hide. When hiding is prevented because of a lack in appropriate resources, the mice may exhibit stereotypic behavior and associated neurophysiological abnormalities. In other instances, a lack of contact with conspecifics in social species may lead to abnormal repetitive behavior, self-injurious behavior, or abnormally exaggerated or blunted responses to commonly occurring stimuli or events in the laboratory (such as handling or the mixing of animals). Distress might also arise from postsurgical pain or an experimental protocol, such as malaise associated with infection or exposure to toxins. Some categories and examples of situations that might be expected to cause distress are shown in Table 1. There are many ways to categorize the sources of distress in the laboratory. The categories listed in Table 1 are not mutually exclusive and may co-occur.

9. Two broad questions might be asked to determine when an animal could be experiencing distress ${ }^{9}$ :

- Is the animal healthy?

- Does the animal have what it wants?

'Wants,' in this context, refers to resources or behaviors that are important to the animal, without which the animal may experience significant unpleasant psychological states and consequently develop or behave abnormally. In human terms, all of the various ways in which distress occurs involve individuals either being in a situation that they want to get out of (and would do so if given the opportunity) or not having something they want (and that they would work for to obtain if they could). For example, distress caused by hunger is reflected operationally as wanting food and being prepared to go to great lengths to get it. Cold-related distress involves wanting to get warmer. Empirical evidence as to the importance an animal places on gaining a resource (or removing itself from a particular situation) provides a basis on which to determine how distressed the animal might be in the absence of this resource (or trapped in that situation).

10. Conditions that compromise animal health or lead to increased risk of injury, disease, or death are often major sources of distress. Moreover, conditions leading to increased mortality, disease, deformity, or injury may cause even greater distress. Evidence that shows the impact of a procedure or husbandry activity on an animal's health or psychological well-being would considerably strengthen any argument that an animal is in distress ${ }^{9}$.

11. A wide range of measures, when considered together, can be used to indicate changes in physiological status and create a picture of the overall health of the animal. These include measures of weight change, and hormonal function (e.g. glucocorticoids), and myriad other clinical signs (see Tables 2 and 3-these tables may serve as examples for inclusion in protocols reviewed by IACUCs). Operational tools also exist for assessing distress that occurs in the absence of physical indicators of ill-health. Some of these measures can be used in situ in the laboratory, while others are useful tools in applied experimental assessment of animal distress. Behavioral measures include:

- Behavioral priorities,

- Demand for resources,

- The presence of abnormal behaviors,

- Reduced behavioral diversity or level of activity compared with healthy control animals,

- The extent of malaise and sickness behavior, and 
- Pain-related behaviors.

Both physiological and behavioral measures can provide information about not only health, but also psychological state. It should be noted that none of these measures, when considered individually, is necessarily sufficient to indicate distress in any given situation. However, a selection of appropriate measures should be considered when assessing the distress associated with particular experimental protocols or husbandry situations.

12. Clinical veterinary methodology is a useful starting point for programs that want to define and mitigate distress. This classical veterinary approach allows the identification of deviations from the normal state, using measurable early signs of overt stress, pain, distress, and ongoing suffering prior to death. This approach also provides a context for evaluating systematic means of intervention.

13. The identification and use of humane endpoints are an important addition to classical veterinary assessments that ensures minimization of later stages of intense distress. Changes in physical health and psychological well-being need to be monitored within the laboratory. One way this can be achieved is through the use of welfare scoring systems such as those described by Morton ${ }^{10}$. Simple, straightforward scoresheets allow efficient welfare and distress evaluation practices and foment rapid intervention when negative changes are detected. Ongoing daily experience, familiarity with the animals, knowledge and awareness of species-typical behavior, and high quality animal diagnostic skills are important in order to effectively identify distress. These qualities will also assist in preventing and/or alleviating subsequent distress in future cases.

TABLE 2. Initial predictive map of a theoretical rat congestive heart failure model. After the consulting veterinary review is summarized or sketched (as in this table), investigators and their veterinary staff may then begin to determine what might be possible to monitor in order to intervene. Table 2 is then expanded to reveal details of detection that will aid in the objective examination (see Table 3).

\begin{tabular}{|c|c|}
\hline Primary Event & Symptoms or Signs \\
\hline Heart failure & $\begin{array}{l}\text { Increased respiratory rate, pallor, tachycardia, } \\
\text { decreased appetite }\end{array}$ \\
\hline Secondary Event & Symptoms or Signs \\
\hline Renal failure, congestion of the liver & $\begin{array}{l}\text { Decreased urine output, enlarged or fluid-distended } \\
\text { abdomen, edema of limbs, diarrhea }\end{array}$ \\
\hline Tertiary Event & Symptoms or Signs \\
\hline Chilling, dehydration, hypoproteinemia & $\begin{array}{l}\text { Piloerection, poor skin, turgor, increased edema, fecal } \\
\text { stains }\end{array}$ \\
\hline
\end{tabular}

14. Definitions of health, such as that advanced by the World Health Organization, emphasize that wellbeing does not occur simply when adverse states are absent ${ }^{11}$. Thus, in addition to asking if the animal is healthy and whether or not it has what it wants, a third question should be asked: Does the animal have the resources it needs to perform those behaviors and social interactions that provide positive experiences? 
An animal's well-being cannot simply be considered the absence of distress; due consideration should be given to designing laboratory environments and husbandry methods which maximize opportunities for animals to perform behaviors that are important to them. These include social behaviors and behaviors that incorporate particular resources, such as a covered shelter that provides places to hide or rest and that have been shown scientifically to have the potential to improve an animal's psychological and physical well-being. Different species may have particular requirements for performing certain behaviors based on their evolutionary history. The importance of performing particular behaviors may also be influenced by an individual's developmental history.

TABLE 3. Expansion of visual and mechanical opportunity to detect and mitigate distress from example in Table 2.

\begin{tabular}{|c|c|c|}
\hline Primary Event & Symptoms or Signs & Diagnostics \\
\hline Heart failure & $\begin{array}{l}\text { Increased respiratory rate, pallor, } \\
\text { tachycardia, decreased appetite }\end{array}$ & Yes, visual exam, weight, scale \\
\hline Secondary Event & Symptoms or Signs & Diagnostics \\
\hline Renal failure, congestion of the liver & $\begin{array}{l}\text { Decreased urine output, enlarged or } \\
\text { fluid-distended abdomen, edema of } \\
\text { limbs, diarrhea }\end{array}$ & $\begin{array}{l}\text { Yes, scale, serum chemistry } \\
\text { analyzer, visual observations }\end{array}$ \\
\hline Tertiary Event & Symptoms or Signs & Diagnostics \\
\hline Chilling, dehydration, hypoproteinemia & $\begin{array}{l}\text { Piloerection, poor skin, turgor, } \\
\text { increased edema, fecal stains }\end{array}$ & $\begin{array}{l}\text { Hematocrit centrifuge, } \\
\text { thermometer or temperature } \\
\text { microchip identification }\end{array}$ \\
\hline
\end{tabular}

\section{RECOMMENDATIONS FOR OVERSIGHT OF ANIMAL RESEARCH AND GOOD PRACTICE}

The workshop developed recommendations that may benefit animal welfare oversight committees and which fall into two broad categories: (1) recommendations for the process and practice of Institutional Animal Care and Use Committees (IACUCs) and others involved in minimizing and preventing animal distress; (2) recommendations for improved availability of resources, training, and evidence to those involved in managing animal distress.

\section{Suggestions for animal welfare oversight activity}

Team approach. The role, responsibility, and contribution of all professionals associated with animal care, including veterinarians, technicians, and principal investigators, should be considered when identifying and resolving issues of animal distress. Moreover, their contributions should be recognized and reflected in decisions regarding the professional development, institutional management, and training of laboratory personnel.

Post-approval monitoring. Retrospective analysis of approved and funded protocols may be beneficial for IACUCs in evaluating the consequences of protocol design and refinement for clinical validity of research, data quality, and prevention/alleviation of animal distress. Where possible, data from such 
analyses should be made available to other IACUCs in order to promote information exchange and consistency, and to demonstrate the costs and benefits of animal-based research. This data could also be used to identify priorities for further work on animal distress.

Research on animal distress. Where data on animal distress is not currently available to assist in decision-making, IACUCs could highlight their priorities for research into the recognition, measurement, and alleviation of pain and distress in animal research to those institutions with regulatory responsibility for minimizing animal distress, such as the USDA and the Office of Laboratory Animal Welfare (OLAW) of the National Institutes of Health.

Professional guidance for IACUCs. IACUCs could seek guidance from specific professional bodies when data on animal distress is unclear. These bodies might include neuroscience societies for neuroscience-related protocols; physiological societies for physiological protocols; the American Veterinary Medical Association (AVMA) for veterinary-related guidance; and the International Society for Applied Ethology (ISAE), Scientists Center for Animal Welfare (SCAW), and Universities Federation for Animal Welfare (UFAW) for advice on applied animal welfare and its assessment. Knowledge of ethological measures related to distress assessment could be enhanced by subscriptions to academic journals such as Applied Animal Behaviour Science, Animal Welfare, and The Journal of Applied Animal Welfare Science. Training and professional development for IACUC members on issues relating to animal distress, through forums such as the Public Responsibility in Medicine \& Research (PRIM\&R) conferences and associated IACUC training, would also be valuable.

\section{Development of resources for IACUCs and researchers}

A range of resources could be developed that would assist IACUCs and others in their efforts to assess and minimize laboratory animal distress. A preliminary list includes, but is surely not limited to, the following:

- Good practices database. A fully searchable database of good practices is needed, detailing a wide range of protocols, validated endpoints, and housing and husbandry techniques. The database should be available electronically.

- Journal of refinement. A scientific journal specifically for publication of refinement articles should be developed.

- Existing journals. It would be beneficial to increase refinement studies and refinement-related protocol details in existing scientific journals, particularly those relating to laboratory animal science. Journals could specifically encourage authors to detail methods used to recognize and minimize pain and distress in submitted papers.

- Keyword searches for refinement. Journal articles should identify key words that facilitate the search for refinements to protocols.

- Primer on pain and distress. Resources such as The HSUS' Pain and Distress Report, which summarizes the latest research and developments pertaining to laboratory animal distress, and the proceedings of this workshop, should be made easily available to IACUCs. Such resources should be widely publicized.

- Research on animal distress. Priorities should be identified for research into the recognition, measurement, funding, and alleviation of pain and distress in animal research. Resources to support such research should be identified and made available.

- Discipline-specific good practice guidelines. Professional bodies for specific academic disciplines and researchers in particular professions (such as neuroscience and physiology) 
should develop good practice guidelines for use by IACUCs to ensure protocol consistency and minimization of animal distress. Veterinary organizations are similarly well placed to provide veterinary guidance on animal welfare.

- Integrated animal welfare and distress assessment. Scientific organizations such as the ISAE and UFAW (and their journals Applied Animal Behaviour Science and Animal Welfare respectively, alongside the Journal of Applied Animal Welfare Science) provide extensive and detailed publications on the recognition, assessment, and minimization of animal distress and could be useful forums for disseminating information on laboratory animal distress. Knowledge of ethological measures related to distress assessment could be increased through the development of training programs for IACUC members. Professional development could include workshops within conferences such as PRIM\&R; dissemination of information and workshops at existing academic conferences, such as that of the ISAE, the American Association for Laboratory Animal Science (AALAS), and the American College of Laboratory Animal Medicine (ACLAM); increased patronage of existing interactive online courses on animal welfare, such as those offered at Cambridge E-Learning Institute (http://www.cambridge-elearning.com), as well as development of similar new programs; and wider IACUC subscription to associated scientific journals, such as Animal Welfare, Applied Animal Behaviour Science, Journal of Applied Animal Welfare Science, and those produced by AALAS.

\section{REFERENCES}

1. USDA APHIS AC. Policy \#11: Painful and Distressful Procedures. USDA Animal and Plant Health Inspection Animal Care Policy Manuel. (1997) http://www.aphis.usda.gov/ac/policy/policy11.html.

2. USDA APHIS. Proposed rule. Animal welfare; definitions for and reporting of pain and distress. Federal Register 65 (132), 42304-42305 (10 July 2000).

3. Plous, S. Attitudes toward the use of animals in psychological research and education: results from a national survey of psychologists. Am. Psychol. 51(11), 1167-1180 (1996).

4. Plous, S. Attitudes toward the use of animals in psychological research and education: results from a national survey of psychology majors. Psychol. Sci. 7, 352-358 (1996).

5. National Science Board. Science and Engineering Indicators - 2002. (National Science Foundation, Arlington, VA, 2002).

6. Stephens, M.L. \& Conlee, K.M. The HSUS'S Pain and Distress Initiative: overview and update. Altern. Lab. Anim. 32(Suppl 1), 423-427 (2004).

7. HSUS. 2000. Taking Animal Welfare Seriously: Minimizing Pain and Distress in Research Animals. (Animal Research Issues Section, The Humane Society of the United States, Washington, DC).

http://www.hsus.org/animals_in_research/pain_distress/taking_animal_welfare_seriously_minimiz min_pain_and_distress_in_research_animals/index.html.

8. National Research Council. Guidelines for the Care and Use of Mammals in Neuroscience and Behavioral Research (National Academies Press, Washington, DC, 2003).

9. Dawkins, M.S. Using behaviour to assess welfare. Anim. Welf. 13(Suppl), S3-S7 (2004).

10. Morton, D.B. A systematic approach for establishing humane endpoints. ILAR J. 41(2), 80-86 (2000).

11. WHO. WHO definition of health. www.who.int/about/definition/en. 